
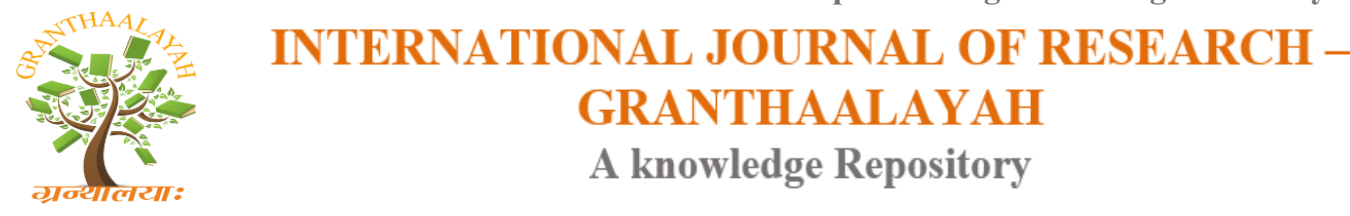

Science

\title{
DETECTION OF CRACKS IN SIMPLY SUPPORTED BEAM BY USING VARIOUS TECHNIQUES: A REVIEW
}

\author{
Tejas Kishor Patil ${ }^{* 1}$, Prof. Ajeet B. Bhane ${ }^{* 2}$ \\ ${ }^{* 1}$ PG Scholar, Department of Mechanical Engineering, SNDCOE\&RC, Yeola, S. P. Pune \\ University, INDIA \\ *2 Assistant Professor, Department of Mechanical Engineering, SNDCOE\&RC, Yeola, S. P. \\ Pune University, INDIA
}

\begin{abstract}
In this review work the crack detection procedure by using vibration and wavelet technique is discussed. Crack converts the linear problem to nonlinear problem and affects the natural frequency. So in this paper we discussed the various methods to find out cracks on beams like destructive testing and non-destructive testing. Wavelet technique is mathematical method alternative to the non-destructive testing like fast fourier transform and is also provides good result compared to other methods. As in this the wavelets are developed and it is solved by various types like mother wavelet technique. This work provides a review for the readers and it is solution to the crack detection by wavelet technique.
\end{abstract}

Keywords:

wavelet technique, nonlinear problems, non-destructive testing, mother wavelet, wavelets.

Cite This Article: Tejas Kishor Patil, and Prof. Ajeet B. Bhane, "DETECTION OF CRACKS IN SIMPLY SUPPORTED BEAM BY USING VARIOUS TECHNIQUES: A REVIEW" International Journal of Research - Granthaalayah, Vol. 3, No. 12(2015): 129-132.

\section{INTRODUCTION}

In mechanical engineering beams are widely used as structural elements and cracks are one of the main source of structural failure and failure analysis is done to promise the safety and economic growth of the industry. In order to reduce or eliminate the sudden failure of structure, they should be regularly and periodically checked for cracks. To localize the inspection and monitoring of damages in structures Non-destructive testing methods such as ultrasonic testing, X-ray, acoustic emission, etc. were adopted. These methods are efficient but laborious, time consuming, expensive and inconvenient for inaccessible structural elements. Based on vibration methods of identifying crack size and location have certain advantages. Wavelet is one of the method which reduces the mistakes. Wavelet based method is proved as fats and inexpensive method for crack detection. In this we have done the literature survey and the survey is written as follow: 
N. G. Jaiswal et al (2015) worked on "Sensitizing the Mode Shapes of Beam towards Damage Detection Using Curvature and Wavelet Transform", the mathematical study was done for damage detection in beam structure with mode shape curvatures and its spatial wavelet transform. Vibration data collected from the perturbed system was processed which were converted into mode shape curvatures and subsequently fed to the wavelet transform. The study reveals that the classical approach based alone on bare modal data was poor in sensitizing damage-induced features than the proposed transformation. It is observed that; by showing relatively larger peaks at the position of the damage decomposition of the spatial signal into wavelet details can identify the damage position in beam like structure ${ }^{[1]}$. Jiawei Xiang et al (2012) worked on "Detect damages in conical shells using curvature mode shape and wavelet finite element method", to form attack damage detection approach to detect locations and severities of damages on the surface of conical shell the damage-induced curvature mode shape and natural frequency changes are taken into consideration in this paper. This approach includes two steps, i.e., the first was to detect damage locations using wavelet transform to decompose a curvature mode shape and the second was to determine damage severities using support vector machine (SVM) to seek from damage severities evaluation database calculated by wavelet-based elements. Particular interest was the damaged conical shell modeled by wavelet-based elements to gain precise displacement mode shape and natural frequencies ${ }^{[2]}$. Wen-Yu He et al (2011) worked on "Finite element analysis of beam structures based on trigonometric wavelet", Taking advantages of trigonometric Hermit wavelet that has both good approximation characteristics of trigonometric function and multi-resolution, local characteristics of wavelet as interpolating function, the trigonometric wavelet finite beam element was formulated by the author to carry out the bending, free vibration and buckling of beam structures. Due to the Hermit interpolation properties of trigonometric wavelet, the proposed trigonometric wavelet finite beam element formulation can deal with the boundary conditions and connection between adjacent elements as the traditional finite element method does. Several numerical examples on the bending, free vibration and buckling analysis of beam structures have demonstrated that the trigonometric wavelet finite element method can achieve a good accuracy with less element adopted, especially for free vibration analysis ${ }^{[3]}$. F. Al-Badour et al (2011) worked on "Vibration analysis of rotating machinery using time-frequency analysis and wavelet techniques", time-frequency analysis including the wavelet transform was one of the new and powerful tools in the important field of structural health monitoring using vibration analysis. Commonly-used signal analysis techniques based on spectral approaches such as the fast Fourier transform were powerful in diagnosing a variety of vibration-related problems in rotating machinery. Although these techniques provide powerful diagnostic tools in stationary conditions they fail to do so in several practical cases involving non-stationary data which could result either from fast operational conditions such as the fast start-up of an electrical motor or from the presence of a fault causing a discontinuity in the vibration signal being monitored. Although the short-time Fourier transform compensates well for the loss of time information incurred by the fast Fourier transform it fails to successfully resolve fast changing signals (such as transient signals) resulting from non-stationary environments. To mitigate this situation wavelet transform tools were considered in this paper as they were superior to both the fast and short-time Fourier transforms in effectively analyzing non-stationary signals ${ }^{[4]}$. Nguyen Viet Khoa et al (2010) worked on "wavelet based technique for multi-crack Detection of a beam-like structure using the vibration data measured directly from a moving vehicle", in this paper an idea for crack detection of a multi-cracked beam-like structure by analyzing the vibration measured directly from the vehicle was presented. The crack 
model was adopted from fracture mechanics. The dynamic response of the bridge- vehicle system was measured directly from the moving vehicle. When the vehicle moves along the structure, the dynamic response of the vehicle was distorted by the cracks at their locations. These distortions were generally small and difficult to be detected visually. Wavelet transform was productive procedure to find out the cracks and to detect such small distortions it was adopted. In the wavelet transform presence of the cracks can be disclosed by large values (peaks). The proposed method is applicable for low velocity-movements while high velocitymovements were not recommended ${ }^{[5]}$. M. Sifuzzaman et al (2009) worked on "Application of Wavelet Transform and its Advantages Compared to Fourier Transform", wavelet analysis was an exciting new method for solving difficult problems in mathematics, physics, and engineering, with modern applications as diverse as wave propagation, data compression, signal conditioning, image handling, pattern recollection, the identification of aircraft and submarines and other medical image technology. Patterns, pictures, voice, songs were decomposed into elementary forms at different positions and scales and subsequently reconstructed with high precision with the help of wavelet ${ }^{[6]}$. Cecilia Surace et al (1994) worked on "Crack Detection of a Beam Using the Wavelet Transform", recognition of the existence of the damage using the computation by the acceleration measuring instrument strategically located on the structure an approach was developed using Wavelet Transform. In detecting relatively small Frequency Response Function to identify the presence of damage the wavelet transform was true. The study described has been undertaken by simulating a cracked cantilever beam using a simple finite element model to provide data for the analysis ${ }^{[7]}$.

\section{CONCLUSION}

Based on this analysis the methods other than conventional methods like Wavelet technique, FFT, Torsional excitation method are successfully used for calculation of damage severity that is the location and crack depth of the beam. FFT is used to find out natural frequencies at different places on beam. By wavelet transform the crack areas are found out based on the remarkable points on the envelope of a specific detail of the decomposed curvature mode shape. Trigonometric Hermit Wavelet is introduced in the field of beam structural analysis. The accuracy of the method is illustrated by case studies involving a short cantilever beam with a crack. A transient excitation is applied for a very short duration at specific angular orientation of the rotor and its effect in the investigation.

\section{ACKNOWLEDGEMENT}

We would like to convey our special gratitude and appreciation towards Prof B. C. Londhe and Prof. A. P. Ghodake for their continuous motivation and valuable assistance in this review work.

\section{REFERENCES}

[1] N. G. Jaiswal, Sensitizing The Mode Shapes Of Beam Towards Damage Detection Using Curvature And Wavelet Transform, International Journal Of Scientific \& Technology Research Volume 4, Issue 04, April 2015.

[2] Jiawei Xiang et al, Detect damages in conical shells using curvature mode shape and 
wavelet finite element method, International Journal of Mechanical Sciences, 2013, 8393.

[3] Wen-Yu He et al Finite element analysis of beam structures based on trigonometric wavelet, Finite Elements in Analysis and Design, Dec. 2011, 59-66.

[4] F. Al-Badour et al (2011) worked on "Vibration analysis of rotating machinery using time- frequency analysis and wavelet techniques, Mechanical Systems and Signal Processing,2013, 2083-2101.

[5] Nguyen Viet Khoa, wavelet based technique for multi-crack Detection of a beam-like structure using the vibration data measured directly from a moving vehicle, Vietnam Journal of Mechanics, VAST, Vol. 32, No. 4 (2010), 222 - 234.

[6] M. Sifuzzaman, Application of Wavelet Transform and its Advantages Compared to Fourier Transform, Journal of Physical Sciences, Vol. 13, 2009, 121-134.

[7] Cecilia Surace et al "Crack Detection of a Beam Using the Wavelet Transform, Research Gate, 1994, 1140-1147. 